\title{
Isolated Sixth Nerve Palsy in Pseudo Gradenigo's Syndrome
}

\author{
Neeta Pal* and Aparna Chakravarthy \\ Karpaga Vinayaga Institute of Medical Sciences, Kanchipuram, India
}

\begin{abstract}
Nasopharyngeal carcinoma is a relatively uncommon malignancy in South India. Exact incidence in Indian population is not known. It usually presents with either epistaxis, mass in the neck, or cranial nerve(s) involvement. This is an interesting case because isolated sixth nerve involvement on presentation is rare.
\end{abstract}

Keywords: Nasopharyngeal carcinoma; Right eye; Ataxia; Left eye

\section{Case Report}

A 71 yrs. old man presented in our hospital with a swelling in the medial canthus of the Right Eye (RE) past 3 months in duration. The swelling was slowly and progressively increasing in size (Figure 1a). It developed induration and ulceration past 1 month. He also complained of right sided periorbital pain. He suffered from serous discharge from his right ear few months back. There was no history of weakness of any part of body, ataxia, or loss of sensation over body. Bladder and bowel habits were normal. There was no history of fever, epistaxis, swellings in neck, haemoptysis, or weight loss. On examination, patient was conscious, and vitals were stable. Examination of chest, cardiovascular system, abdomen and central nervous system was normal. There were no other systemic or neurologic symptoms. There was no history of smoking or drug/alcohol abuse. His best corrected visual acuity in RE was 20/70 and in Left Eye (LE) was 20/30. Ocular motility test showed RE abduction deficit (Figure 1b) while movements were preserved on the left side (Figure 1c). Thus diplopia was present in right lateral gaze. Pupils were bilaterally equal and normally reacting to light. Clinically it was chronic otitis media presenting with Gradenigos syndrome. The patient was further sent for radiological investigations to confirm the diagnosis.

Computed Tomography (CT) (Figure 2) findings were suggestive

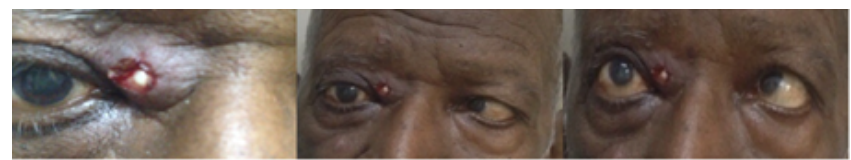

Figure 1: (a) Medical canthal ulcerating mass. (b) RE: Abduction deficit. (c) No restrictions in other gazes.

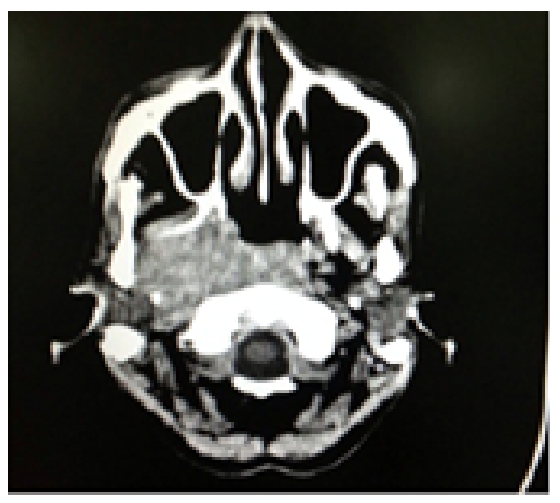

Figure 2: CT findings: Nasopharyngeal carcinoma of the right side with extension into both sinuses, right middle ear cavity, right temporo-mandibular joint and mass effect and edema in right temporoparietal region. of ill-defined soft tissue mass $(6.9 \times 6.8 \times 5.6) \mathrm{cm}$ in size in the right nasopharynx with extensions upto the angle of mandible of right side, above up to the base of skull, right cavernous sinus and bilateral sphenoid sinuses. There was lytic destruction with bone rarefaction involving clivus, squamous part of right temporal bone, walls of both sphenoid sinuses and soft tissue extension into both sinuses, right middle ear cavity, right temporo-mandibular joint. The lesion crosses the midline to the left side causing lytic destruction of condylar process in the base of skull with possible mass effect at cervico-medullary junction and right temporoparietal region. CT with contrast was done to confirm these findings. Therefore, the final diagnosis was nasopharyngeal carcinoma leading to 6th nerve palsy of right side. The triad of Gradenigo's syndrome when presents with nasopharyngeal carcinoma is called Pseudo Gradenigo's syndrome. Audiometry test showed sensorineural hearing loss of both ears.

He was treated with combination chemotherapy with 5-fluorouracil and cisplatinum alongwith external beam radiotherapy. The patient was given one cycle of chemotherapy and radiotherapy, and is yet to report back for follow-up.

\section{Discussion}

In 1904 Guiseppe Gradenigo described an infection of the apex of the petrous part of the temporal bone from acute otitis media with the clinical symptoms of unilateral pain around the eye, diplopia due to sixth nerve paralysis and persistant otorrhea [1]. Contact with the tip of the petrous pyramid makes the portion of the sixth nerve within Dorello's canal susceptible to pathologic processes. This is especially important in cases secondary to localized inflammations or extradural abscesses due to complicated otitis media. Nasopharyngeal carcinoma may also mimic Gradenigo's syndrome, since it can obstruct the Eustachian tube, resulting in serous otitis media. Additionally, the carcinoma may subsequently invade the cavernous sinus, causing sixth nerve paresis [2]. Therefore, its called as Pseudo-Gradenigo syndrome.

Nasopharyngeal carcinoma is the most dreaded etiology of sixth nerve palsy and most frequently occurs between the ages of 40 and 70 years. It may present with various symptoms including nasal obstruction, rhinorrhea, epistaxis and serous otitis media secondary to obstruction of the Eustachian tube. This tumour may also involve the

*Corresponding author: Neeta Pal, Karpaga Vinayaga Institute of Medical Sciences, Kanchipuram, India, Tel: 044-27565195; E-mail: neeta.pal1809@gmail.com

Received August 13, 2015; Accepted August 22, 2015; Published August 29 2015

Citation: Pal N, Chakravarthy A (2015) Isolated Sixth Nerve Palsy in Pseudo Gradenigo's Syndrome. J Clin Case Rep 5: 582. doi:10.4172/2165-7920.1000582

Copyright: ( 2015 Pal N, et al. This is an open-access article distributed under the terms of the Creative Commons Attribution License, which permits unrestricted use, distribution, and reproduction in any medium, provided the original author and source are credited. 
Citation: Pal N, Chakravarthy A (2015) Isolated Sixth Nerve Palsy in Pseudo Gradenigo's Syndrome. J Clin Case Rep 5: 582. doi:10.4172/21657920.1000582

cranial nerves in many ways. Firstly, the tumor may extend superiorly through foramen lacerum, which is an unimpeded pathway near the fossa of Rosenmuller into the cranium [3-5], thus involving the cranial nerves in the middle cranial fossa and cavernous sinus. In our patient, the extension of the tumor into cavernous sinus lead to sixth nerve palsy without involvement of other cranial nerves. Sixth nerve is the second most common nerve to be involved in nasopharyngeal carcinoma. Isolated sixth nerve palsy, a rare neuro-ophthalmologic presentation for this tumor [6]. Only one other case has noted isolated sixth nerve palsy [7]. The sphenoid sinus is also frequently involved by superior extension of the tumour $[8,9]$. Similarly in our patient extensive extension of the tumor was noted.

\section{References}

1. Gradenigo G (1904) A special syndrome of endocranial otitic complications (paralysis of the motor oculi externus of otitic origin). Ann Otol 13: 637-639.

2. Reinhardt F, Lange E (1997) The vascular cerebellopontile angle syndrome. Psychiatr Neurol Med Psycho 29: 266-274.
3. Takami T, Ohata K, Tsuyuguchi N, Mao Y, Inoue Y, et al. (2002) Cavernous sinus metastasis from thyroid papillary adenocarcinoma. J Clin Neurosci 9 : 598-600.

4. Dare AO, Gibbons KJ, Proulx GM, Fenstermaker RA (2003) Resection followed by radiosurgery for advanced juvenile nasopharyngeal angiofibroma: report of two cases. Neurosurgery 52: 1207-1211.

5. Ablashi DV, Levine PH, Pearson GR (1983) Meeting report: Fourth International Symposium on Naso-pharyngeal Carcinoma Application of Field and Laboratory Studies to the Control of NPC. Cancer Res 43: 2375-2378.

6. Hara HJ (1969) Cancer of the nasopharynx: Review of the literature; report of 72 cases. Laryngoscope 79: 1315-1329.

7. Neel HB III, Taylor WF (1983) Clinical presentation and diagnosis of Nasopharyngeal Carcinoma: Current status Naso-pharyngeal Carcinoma Current concepts In Prasad U Kuala Lumpur, Malaysia.

8. Jane WC (2003) Sixth nerve palsy in nasopharyngeal carcinoma. Neurology 61: 1417.

9. Savino PJ, Hilliker JK, Casell GH (1982) Chronic sixth nerve palsies. Arch Ophthalmol 100: 1442-1444. 